AN AUDIENCE WITH...

\section{David Liu}

If drug discovery is the art of taking smart risks, then David Liu is a virtuoso.

A Harvard chemical biologist who has been kicked out of casinos for winning too much money with a blackjack card-counting system of his own creation, Liu has developed genome-editing tools that may one day be turned into therapeutics and DNA-encoded libraries that can help in the hunt for small-molecule drug leads. His attitude towards risk - and its minimization - has also led him to become a serial biotech entrepreneur, setting up six biotech firms, including Ensemble Therapeutics, Editas Medicine, Beam Therapeutics and another company that is in the works. He spoke with Asher Mullard about the future of genome editing, the influence of DNA-encoded libraries and how company creation has influenced his career.

\section{Q Do you consider yourself to be a gambler and has that influenced your approach to science? \\ While card counting is technically gambling, it's an atypical form of gambling because you play at a mathematical advantage. And I see analogies to the kinds of risks and potential pay-offs that we encounter every day as researchers, in that the problems that we work on are by definition ones where we don't know in advance what the outcomes will be. All we can do is try to understand the phenomenon as deeply as possible in the hopes of stacking the odds in our favour. \\ But the less romantic truth is that I've always picked research problems led purely by my curiosity, by what I believe to be the most interesting and most important problems that I can work on.}

\section{Q When and how did CRISPR genome-} editing technologies pique your curiosity? My interest in being able to programmably manipulate the genome began with a project I started in 1999, called 'the Unifactor 2000'. Anybody reading this article who was involved in this project is probably getting post-traumatic stress disorder right now. The point of this project was to create a universal transcription factor, using DNA triplex formation or DNA-DNA-RNA triplex formation to programmably target different sites in the genome with transcriptional activators, silencers or nucleases that could turn on, turn off or cut those sites. And the project utterly failed because triplex formation in cells turns out to not be a very general phenomenon. At least that's why I believe it failed.
When zinc fingers and then TALENs and eventually CRISPR-Cas9 came onto the scene, opportunities to restart thinking about programmable genome manipulation emerged. And I again became very passionate and curious about what we could achieve if we could address the genome in a programmable way. And nothing about this renewed interest was a remarkable insight on my part; many other researchers were also waiting for a really good, robust way to programme the genome.

While it's still not quite accurate to say that we can bring anything to any site in the genome in any living cell or organism, we can currently cover a good fraction of it.

Q. Agenome-editing gold rush is underway, and researchers are using genome mining, engineering and evolutionary strategies to find the next generation of newer and better editing tools. What's your strategy?

Our main area of research within genome editing is base editing. Our interest in base editing stemmed from the simple realization that most mutations that cause human genetic diseases are point mutations,

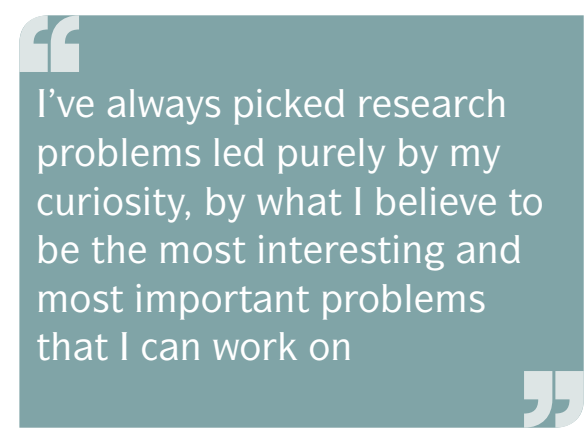

but current standard genome-editing methods including CRISPR-Cas9 work by cutting DNA, which doesn't result in fixing point mutations most of the time.

The first class of base editors that we developed turned out to require a lot of engineering. These base editors use the targeting mechanism of CRISPR nucleases to home in on a single location in the genome, but instead of cutting the DNA, these base editors convert one base into another base. We engineered our first class of base editor from three separate proteins. First, we disabled the ability of CRISPR nucleases to cut DNA while retaining their ability to bind DNA in a programmable manner. Second, we linked those disabled CRISPR scissors to cytidine deaminase enzymes, which transform $\mathrm{C}$ into a base that resembles T. Third, we connected this to a protein that prevents the cell from removing the edited base and turning it back into a C. And then of course we had to make base editing efficient and permanent by fixing the other strand as well, and we realized that we could further engineer our three part proteins to flag the non-edited partner DNA strand for replacement by nicking it, tricking the cell into changing the $\mathrm{G}$ on the non-edited strand into an $\mathrm{A}$.

That tool enables the conversion of Cs into Ts, and Gs into As. These two changes in theory can correct up to $14 \%$ of known pathogenic point mutations. But correcting the largest fraction of mutations that cause genetic disease required the development of a second class of base editor that converts As into Gs and Ts into Cs. Those could in theory correct almost half of known pathogenic point mutations. 
For that second class of base editors, we again used the targeting mechanism of disabled CRISPR scissors to localize the base editor to the right position in the genome. But we ran into this huge problem in that there is no naturally occurring enzyme to convert As into Gs or Ts into Cs. That's where the evolution came in; we decided that we would actually try to evolve in the laboratory our own enzymes to perform this chemical reaction. It was a pretty ambitious plan, and I give a lot of credit to a former post doc in my lab Nicole Gaudelli and her co-workers for having the courage to proceed. After years of effort, we succeeded in evolving the first enzyme known to convert an A into a base that looks like a G. That eventually became the business end of the second base editor.

\section{Q. What kind of other editing tools and optimizations are still needed in this space?} The ability to change any DNA sequence into any other DNA sequence is one of the grand challenges of the life sciences. And there's still a long road ahead. Delivering genome-editing agents into the human body remains difficult in many cases, so we need to continue to improve delivery strategies. And then of course on the actual editing machinery side, we need to continue to find ways to perform all the remaining ways of changing one base pair to another. The two base editors that we've developed so far collectively can make changes that in principle can correct up to about $62 \%$ of pathogenic point mutations, but that leaves thousands of point mutations that we can't fix. We also have to keep working to maximize the efficiency of editing, and to minimize unwanted editing in off-target locations. So there's still a lot of work ahead.

\section{Q Beam Therapeutics, which you co-founded to advance base pair editors, is also working with RNA editors. How do these fit into the picture? \\ Beam Therapeutics has licensed the rights to Feng Zhang's RNA-editing technology, and is exploring the possibility of editing RNA rather than DNA at the single-letter level. And there are some situations where making a change in the RNA might offer advantages over changing DNA, such as when you want a transient therapy rather than a permanent one.}

Q What do you make of concerns around off-target editing with base editors? Minimizing off-target editing is very important, but I don't believe that it will ultimately make or break genome-editing agents in the clinic. The first couple of waves of these genome-editing therapeutics will treat diseases that are so grievous that the potential clinical benefits will far outweigh the risks. And ongoing - and quite effective - efforts are already published or underway to minimize off-target editing risks.

But I'd also add that the genome isn't static anyway, and every day the cells in your body collectively accumulate billions of mutations. For example, roughly 300 times a day in every one of your cells a $\mathrm{C}$ becomes a $\mathrm{U}$ in your genome, and if those aren't fixed then that causes the C-to-T mutation. That's the classic mutation that our A-to-G base editor reverses, by the way. Spontaneous mutation frequencies put off-target editing rates in perspective.

None of this is to say that we should dismiss the importance of preventing off-target mutations. But we should at least view off-target editing as a bounded problem. It's not as though we forever need to always go lower and lower. And no therapeutic in the history of therapeutics has ever been perfect and free of side effects.

\section{Q You started working on DNA-encoded libraries (DELs), a means of generating and cataloguing huge numbers of small molecules, nearly 20 years ago. How has it} felt to watch this field mature?

It's been terrific to see how DELs have become just part of mainstream smallmolecule discovery. Virtually every major pharmaceutical company and even a number of academic labs are now using DELs. This is quite a contrast from the first responses I got to my grant proposals for funding for our earliest DEL work, which were rather skeptical that DELs would ever be useful.

In our lab, we continue to generate new DELs and to use them to discover really interesting biological phenomena. In an upcoming Nature Chemical Biology article, for example, we used a DEL to find a small molecule that can actually reprogramme the substrate selectivity of a therapeutically relevant enzyme. And we just founded a new company called Exo Therapeutics, still in stealth mode, that is going to progress this work by developing a generalizable platform to discover drugs that don't just turn off their targets, but instead reprogramme their activities in more sophisticated ways.
Q. Despite widespread industry adoption of $D E L s$, there are only a few public examples of clinical candidates that came from DEL hits. What does this say about the technology?

It's really hard to keep track of exactly what molecules came from DELs. And this is partly because DEL hits can serve as optimization starting points, or as probes that result in the discovery of new molecules that aren't discovered in DELs. But you know I view this as a sign of the healthy maturation of the technology, where people are no longer fixated on the fact that a compound came out of a DEL and instead just view the technology as one important part of the broader drug discovery process.

\section{Q Ensemble Therapeutics, the first company that you created from your work in this space, folded in 2017. Why did it fail?}

I think in retrospect that Ensemble was a bit before its time. It was one of the first, if not the first, DEL company. And it was the first company I founded. I learned a lot just from watching the impact of different business decisions and scientific decisions that together determined the fate of the company though, and it was an invaluable learning experience.

Q You've since set up five more companies. When you started out in science, did you plan on becoming a serial entrepreneur? No not at all. If you had asked me when I was just starting as a professor how many companies I thought I would found during my career, I would have guessed zero.

\section{Q Has this experience shaped your career?} It has been very helpful and very perspectivebuilding. It's pretty easy in academia to work on a problem with a relentless focus in a way in which you risk becoming disconnected from what the rest of the world really needs. Maintaining a connection to other scientists and to the users of science outside of academia is a vital part of building the scientific perspective.

I've had the very good fortune that in Boston interesting science rapidly attracts the interest of investors and others who want to spend the next phase of their lives advancing science to benefit society. And I now view contributing to that system as an obligation. Our research is supported by taxpayer money and we write papers and give talks about the potential of our research to benefit society, so I think we should do what we can to actually facilitate that transition. 